\title{
MULTIBASIC EULERIAN POLYNOMIALS
}

\author{
DOMINIQUE FOATA AND DORON ZEILBERGER
}

\begin{abstract}
Eulerian polynomials with several bases are defined. Their combinatorial interpretations are given as well as congruence properties modulo some ideals generated by cyclotomic polynomials.
\end{abstract}

\section{INTRODUCTION}

It is well known (see, e.g., [Ri, Chapter 7]) that the identity

$$
\sum_{n} \frac{u^{n}}{n !} A_{n}(t)=\frac{1-t}{1-t \exp ((1-t) u)} \quad(n \geq 0)
$$

defines a sequence of polynomials $\left(A_{n}(t)\right)$, called Eulerian polynomials, whose classical combinatorial interpretation is the following: for each permutation $\sigma=\sigma(1) \cdots \sigma(n)$ let des $\sigma$ be the number of descents of $\sigma$, that is, the number of $i$ such that $1 \leq i \leq n-1$ and $\sigma(i)>\sigma(i+1)$. Then $A_{n}(t)=\sum_{\sigma} t^{1+\operatorname{des} \sigma}$ $\left(\sigma \in \mathscr{S}_{n}\right)$. This shows, in particular, that each polynomial $A_{n}(t)$ has positive integral coefficients whose sum is $n$ !.

As usual, let

$$
(u ; q)_{n}= \begin{cases}1, & \text { if } n=0 \\ (1-u)(1-u q) \cdots\left(1-u q^{n-1}\right), & \text { if } n \geq 1\end{cases}
$$

denote the $q$-ascending factorial and also let

$$
(u ; q)_{\infty}=\prod_{n=0}^{\infty}\left(1-u q^{n}\right) .
$$

Further, let

$$
\left[\begin{array}{c}
n \\
i
\end{array}\right]_{q}=\frac{(q ; q)_{n}}{(q ; q)_{i}(q ; q)_{n-i}}
$$

Received by the editors October 23, 1989.

1980 Mathematics Subject Classification (1985 Revision). Primary 05A05, 05A15, 05A30, 11B65, 11B68; Secondary 20C30.

Key words and phrases. Tableaux, major index, descents, permutation statistics, bijective proofs, sign reversing involutions, Robinson-Schensted correspondence.

The first author was supported in part by NSF grant DMS 8901610. On leave (1989) from Département de Mathématique, Université Louis-Pasteur, 7, Rue René-Descartes, F-67084 Strasbourg, France.

The second author was supported in part by NSF grant DMS 8800663 . 
denote the Gaussian polynomial for $0 \leq i \leq n$. Finally, let the two $q$ exponentials be given by (see [An, Chapter 2])

$$
\begin{gathered}
e(u ; q)=\sum_{n \geq 0} \frac{u^{n}}{(q ; q)_{n}}=(u ; q)_{\infty}^{-1} ; \\
E(u ; q)=\sum_{n \geq 0} q^{n(n-1) / 2} \frac{u^{n}}{(q ; q)_{n}}=(-u ; q)_{\infty} .
\end{gathered}
$$

Our purpose is to investigate the properties of $\left(A_{\mathbf{n}}\left(s ; q_{1}, \ldots, q_{l}\right)\right) \quad\left(\mathbf{n}=\left(n_{1}, \ldots\right.\right.$, $\left.\left.n_{l}\right) \in \mathbf{N}^{l}, l \geq 1\right)$ a class of polynomials in $(l+1)$ variables [in short, $A_{\mathbf{n}}(s ; \mathbf{q})$ ] defined by the identity

$$
\sum_{\mathbf{n}} \frac{\prod_{j} u_{j}^{n_{j}}}{\prod_{j}\left(q_{j} ; q_{j}\right)_{n_{j}}} A_{\mathbf{n}}(s ; \mathbf{q})=\frac{1-s}{1-s \prod_{j} e\left((1-s) u_{j} ; q_{j}\right)},
$$

where $j$ goes from 1 to $l$. To convince ourselves that each $A_{\mathbf{n}}(s ; \mathbf{q})$ is a polynomial with integral coefficients we may rewrite $(1.4)$ as $L=(1-R)^{-1}$. The identity $L(1-R)=1$ yields the recurrence relation

$$
A_{\mathbf{n}}(s ; \mathbf{q})=\sum_{\mathbf{i}} \prod_{j}\left[\begin{array}{l}
n_{j} \\
i_{j}
\end{array}\right]_{q_{j}} A_{\mathbf{i}}(s ; \mathbf{q}) s(1-s)^{n_{1}-i_{1}+\cdots+n_{l}-i_{l}-1},
$$

where $j$ goes from 1 to $l$ and where $\mathbf{i}=\left(i_{1}, \ldots, i_{l}\right)$ runs over all sequences of nonnegative integers satisfying $\mathbf{i} \neq\left(n_{1}, \ldots, n_{l}\right)$ and $0 \leq i_{1} \leq n_{1}, \ldots, 0 \leq$ $i_{l} \leq n_{l}$. As $A_{\mathbf{0}}(s ; \mathbf{q})=1$, relation $(1.5)$ shows by induction that each $A_{\mathbf{n}}(s ; \mathbf{q})$ is a polynomial with integral coefficients. To show that the coefficients are indeed positive and of sum $\left(n_{1}+\cdots+n_{l}\right)$ ! requires more analysis and will be a consequence of Theorem 1 or Theorem 2 below.

The second class of polynomials under study will be denoted by $\left(B_{\mathbf{n}}(s ; \mathbf{q})\right)$ $\left(\mathbf{n} \in \mathbf{N}^{k+1}, k \geq 0, \mathbf{q}=\left(q_{1}, \ldots, q_{k}\right)\right)$. They are defined by

$$
\begin{aligned}
\sum_{\mathbf{n}} & \frac{\prod_{j=1}^{k+1} u_{j}^{n_{j}}}{\prod_{j=1}^{k}\left(q_{j} ; q_{j}\right)_{n_{j}} n_{k+1} !} B_{\mathbf{n}}(s ; \mathbf{q}) \\
& =\frac{1-s}{1-s \prod_{j=1}^{k} e\left((1-s) u_{j} ; q_{j}\right) \exp \left((1-s) u_{k+1}\right)} .
\end{aligned}
$$

Assume $0 \leq k, k+1=l$ and let $q_{l}=1$ in (1.5). Then $A_{\mathbf{n}}(s ; \mathbf{q})$ is transformed into a polynomial in $(k+1)$ variables $s, q_{1}, \ldots, q_{k}$. Furthermore, the Gaussian polynomial $\left[\begin{array}{l}n_{l} \\ i_{l}\end{array}\right]_{q_{l}}$ becomes the ordinary binomial coefficient $\left(\begin{array}{l}n_{l} \\ i_{l}\end{array}\right)$. Hence (1.4) itself is transformed into identity (1.6). Thus

$$
B_{n_{1}, \ldots, n_{k+1}}\left(s ; q_{1}, \ldots, q_{k}\right)=\left.A_{n_{1}, \ldots, n_{k+1}}\left(s ; q_{1}, \ldots, q_{k+1}\right)\right|_{q_{k+1}=1} .
$$

Conversely, put $u_{k+1}=0$ in (1.6) and let $k=l$. Then (1.6) yields (1.4), so that

$$
A_{n_{1}, \ldots, n_{k}}\left(s ; q_{1}, \ldots, q_{k}\right)=B_{n_{1}, \ldots, n_{k}, 0}\left(s ; q_{1}, \ldots, q_{k}\right) .
$$


We emphasize the fact that $A_{n}(s ; \mathbf{q})$ has $l$ indices and $(l+1)$ variables, whereas $B_{\mathbf{n}}(s ; \mathbf{q})$ has $(k+1)$ indices and $(k+1)$ variables.

When $k=0$, then $B_{\mathbf{n}}\left(s, q_{1}, \ldots, q_{k}\right)$ reduces to the usual Eulerian polynomial $A_{n}(s)$ defined in $(1.1)$. When $l=1$, then $A_{\mathrm{n}}\left(s, q_{1}, \ldots, q_{l}\right)$ is the $q$-Eulerian polynomial $A_{n}(s, q)$ that is known to be [St] the generating polynomial for the permutation group by the bivariate statistic $(1+$ number of descents, inversion number). Identity (1.6) for $k=1$ was considered by Désarménien [De] for his definition of the q-Eulerian polynomials with two indices.

The theory of basic hypergeometric series is now well understood witness the forthcoming book by Gasper and Rahman [GaRa2]. They have also paved the way to the almost untouched forest of multi-basic hypergeometric series [Ga, GaRa1]. Our intention here is to derive the properties of the multibaseanalog of the classical Eulerian polynomials using mainly symmetric function techniques and combinatorial manipulations on the inversion numbers.

We first give a combinatorial interpretation to $A_{\mathbf{n}}(s ; \mathbf{q})$ and $B_{\mathbf{n}}(s ; \mathbf{q})$. Because of (1.7) it suffices to do it for the $A_{\mathbf{n}}(s ; \mathbf{q})$ 's. Two combinatorial interpretations will be given, the first one in terms of lignes of route of permutations, the second one in terms of inversion numbers. The ligne of route Ligne $\sigma$ of $\sigma=\sigma(1) \cdots \sigma(n)$ is defined to be the set of all $r$ such that $1 \leq r \leq n-1$ and $\sigma(r)>\sigma(r+1)$. Hence $\mid$ Ligne $\sigma \mid$ is the number of descents, $\operatorname{des} \sigma$, defined above. Furthermore, the sum of all $r$ in Ligne $\sigma$ is the classical major index, maj $\sigma$, of $\sigma$.

Let $\mathbf{n}=\left(n_{1}, \ldots, n_{l}\right)$ satisfy $n_{1}+\cdots+n_{l}=n$. Also let $n_{0}=0$ and denote by $\mathbf{n}_{j}$ the partial sum $\mathbf{n}_{j}=n_{1}+\cdots+n_{j}(0 \leq j \leq l)$. For $j=1, \ldots, l$ define the $j$ th $\mathbf{n}$-inverse ligne of route of $\sigma$ as being

$$
\text { Iligne }_{j} \sigma=\left\{r: \mathbf{n}_{j-1}+1 \leq r \leq \mathbf{n}_{j}-1, \sigma^{-1}(r)>\sigma^{-1}(r+1)\right\},
$$

and the $j$ th $\mathbf{n}$-inversion number of $\sigma$ as

$$
\operatorname{inv}_{j} \sigma=\#\left\{\left(r, r^{\prime}\right): \mathbf{n}_{j-1}+1 \leq r<r^{\prime} \leq \mathbf{n}_{j}, \sigma^{-1}(r)>\sigma^{-1}\left(r^{\prime}\right)\right\} .
$$

Also let

$$
\operatorname{imaj}_{j} \sigma=\sum\left\{r-\mathbf{n}_{j-1}: r \in \text { Iligne }_{j} \sigma\right\} \quad(j=1, \ldots, l) .
$$

When $l=1$, the statistics $\operatorname{imaj}_{1} \sigma$ and inv $_{1} \sigma$ are the familiar inverse major index and inversion number of the permutation $\sigma$, respectively.

The first two results of the paper can be stated as follows.

Theorem 1. For each $\mathbf{n}=\left(n_{1}, \ldots, n_{l}\right)$ the generating polynomial for the permutation group $\mathscr{S}_{n}$ by the $(l+1)$-vector $\left(1+\right.$ des, imaj $_{1}, \ldots$, imaj $\left._{l}\right)$ is equal to $A_{\mathbf{n}}(s ; \mathbf{q})$. In other words, if $A_{\mathbf{n}}(s ; \mathbf{q})$ is defined by identity (1.4), then

$$
A_{\mathbf{n}}(s ; \mathbf{q})=\sum_{\sigma} s^{1+\operatorname{des} \sigma} q_{1}^{\text {imaj }_{1} \sigma} \cdots q_{l}^{\text {imaj }_{l} \sigma} \quad\left(\sigma \in \mathscr{S}_{n}\right) .
$$


Theorem 2. For each $\mathbf{n}=\left(n_{1}, \ldots, n_{l}\right)$ the generating polynomial for the permutation group $\mathscr{S}_{n}$ by the $(l+1)$-vector $\left(1+\mathrm{des}, \mathrm{inv}_{1}, \ldots, \mathrm{inv}_{l}\right)$ is equal to $A_{\mathbf{n}}(s ; \mathbf{q})$, i.e.,

$$
A_{\mathbf{n}}(s ; \mathbf{q})=\sum_{\sigma} s^{1+\operatorname{des} \sigma} q_{1}^{\text {inv }_{1} \sigma} \cdots q_{l}^{\text {inv } \sigma} \quad\left(\sigma \in \mathscr{S}_{n}\right) .
$$

The proof of Theorem 1, derived in $\S 2$, makes use of symmetric function techniques, especially the Schur function algebra. To prove Theorem 2 it is more convenient to show that $A_{\mathbf{n}}(s ; \mathbf{q})$, as defined in (1.10) satisfies the recurrence relation (1.5). This is done in $\S 3$, by using an appropriate factorization of permutations.

The combination of (1.9) and (1.10) suggests that there must be a bijection of $\mathscr{S}_{n}$ onto itself that sends the vector $\left(1+\right.$ des, imaj $_{1}, \ldots$, imaj $\left._{l}\right)$ onto $(1+$ des, $\left.\operatorname{inv}_{1}, \ldots, \operatorname{inv}_{l}\right)$. Such a bijection can indeed be derived (§4). Call it $\sigma \mapsto$ $\sigma^{\prime}$. It has even the following stronger property.

Theorem 3. The bijection $\sigma \mapsto \sigma^{\prime}$ satisfies:

$$
\text { Ligne } \sigma=\operatorname{Ligne} \sigma^{\prime} ; \quad \operatorname{imaj}_{j} \sigma_{j}=\operatorname{iriv}_{j} \sigma^{\prime} \quad(j=1, \ldots, l) .
$$

In particular, $\operatorname{des} \sigma=\operatorname{des} \sigma^{\prime}$ and $\operatorname{maj} \sigma=\operatorname{maj} \sigma^{\prime}$.

In its turn Theorem 3 suggests that the polynomial

$$
{ }^{1} A_{\mathbf{n}}(s, p ; \mathbf{q})=\sum_{\sigma} s^{\operatorname{des} \sigma} p^{\text {maj } \sigma} q_{1}^{\text {imaj }_{1} \sigma} \cdots q_{l}^{\text {imaj }_{l} \sigma} \quad\left(\sigma \in \mathscr{S}_{n}\right)
$$

should also be investigated. Using the techniques developed in [DeFo2] its generating function can be calculated in the form

$$
\sum_{\mathbf{n}} \frac{\prod_{j} u_{j}^{n_{j}}}{(s ; p)_{n_{1}+\cdots+n_{l}} \prod_{j}\left(q_{j} ; q_{j}\right)_{n_{j}}}{ }^{1} A_{n}(s, p ; \mathbf{q})=\sum_{r} \frac{s^{r}}{\prod_{j}\left(u_{j} ; p, q_{j}\right)_{r+1, \infty}},
$$

where

$$
\left(u_{j} ; p, q_{j}\right)_{r+1, \infty}=\prod_{1 \leq i \leq r+1}\left(u_{j} p^{i-1} ; q_{j}\right)_{\infty} \quad(j=1, \ldots, l) .
$$

However the series on the right-hand side cannot be put into a form explicit enough to deserve further study. Note that the previous generating function for the ${ }^{1} A_{n}(s, p ; \mathbf{q})$ 's specializes for $l=1$ to an identity worked out by Gessel [Ge1]. For $p=1$ the right-hand side can be summed, as will be shown in the proof of Theorem $1(\S 2)$. The idea of having the ligne of route of a permutation split into compartments is due originally to Remmel [Re]. Further work along these lines can be found in [DeFo2].

The second goal of the paper is to work out congruence properties for the polynomials $B_{\mathbf{n}}(s ; \mathbf{q}) \quad\left(\mathbf{n} \in \mathbf{N}^{k+1}\right)$. Let $\left(d_{1}, \ldots, d_{k}\right)$ be a given sequence of positive integers and for each $\mathbf{n}=\left(n_{1}, \ldots, n_{k}, n_{k+1}\right)$ let

$$
n_{j}=d_{j} a_{j}+b_{j}, \quad 0 \leq b_{j} \leq d_{j}-1 \quad(1 \leq j \leq k),
$$


be the Euclidean division of $n_{j}$ by $d_{j}$. Then define div $\mathbf{n}$ to be

$$
\operatorname{div} \mathbf{n}=\left(b_{1}, \ldots, b_{k}, n_{k+1}+a_{1}+\cdots+a_{k}\right) \text {. }
$$

Further let $\Phi_{d}(q)$ denote the $d$ th cyclotomic polynomial $\left(\Phi_{1}(q)=1-q\right.$, $\left.\Phi_{2}(q)=1+q, \ldots\right)$. We also prove the following theorem.

Theorem 4. We have the congruences

$$
\begin{aligned}
B_{\mathbf{n}}(s ; \mathbf{q}) \equiv(1-s)^{\left(d_{1}-1\right) a_{1}+\cdots+\left(d_{k}-1\right) a_{k}} B_{\operatorname{div} \mathbf{n}}(s ; \mathbf{q}) & \left(\bmod \left(\Phi_{d_{1}}\left(q_{1}\right), \ldots, \Phi_{d_{k}}\left(q_{k}\right)\right)\right) .
\end{aligned}
$$

The case $k=1$ is due originally to Désarménien [De] (see also [DeFo2]).

Corollary. If all the remainders $b_{j}$ in (1.11) are equal to 0 or 1 , then

$$
\begin{aligned}
B_{\mathbf{n}}(s ; \mathbf{q}) \equiv(1-s)^{\left(d_{1}-1\right) a_{1}+\cdots+\left(d_{k}-1\right) a_{k}} A_{a_{1}+b_{1}+\cdots+a_{k}+b_{k}+n_{k+1}}(s) & \left(\bmod \left(\Phi_{d_{1}}\left(q_{1}\right), \ldots, \Phi_{d_{k}}\left(q_{k}\right)\right)\right) \\
&
\end{aligned}
$$

$$
\begin{aligned}
A_{\mathbf{n}}(s ; \mathbf{q}) \equiv(1-s)^{\left(d_{1}-1\right) a_{1}+\cdots+\left(d_{l}-1\right) a_{l}} A_{a_{1}+b_{1}+\cdots+a_{l}+b_{l}}(s) & \\
& \left(\bmod \left(\Phi_{d_{1}}\left(q_{1}\right), \ldots, \Phi_{d_{k}}\left(q_{k}\right)\right)\right) ;
\end{aligned}
$$

where the polynomial $A_{a_{1}+\ldots}(s)$ on both right-hand sides is the Eulerian polynomial defined in (1.1).

In particular, if $\mathbf{n}=\left(2 a_{1}+b_{1}, \ldots, 2 a_{l}+b_{l}\right)$ with $b_{1}=0,1, \ldots, b_{l}=0,1$ and $q_{1}=\cdots=q_{l}=q$, then

$$
A_{\mathbf{n}}(s ; \mathbf{q}) \equiv(1-s)^{a_{1}+\cdots+a_{l}} A_{a_{1}+b_{1}+\cdots+a_{l}+b_{l}}(s) \quad(\bmod (1+q)) .
$$

Both Theorem 4 and its corollary are proved in $\S 5$. The case $l=1$ of $(1.14)$ was in fact conjectured by Loday [Lod] for a side calculation of the cyclic homology of commutative algebras. It has since received several specific proofs in [DeFo2 and Wa]. Michelle Wachs [Wa] derived a very ingenious involution for proving $(1.14)$ in the case $l=1$. In $\S 6$ we give a Wachs-style combinatorial proof of Theorem 4.

\section{Proof of TheOREM 1}

In fact (1.4) with the interpretation given in (1.7) can be viewed as a specialization of a result of the $(k, l)$-colored permutations derived in [DeFo2]. To make the paper self-contained we have preferred to prove Theorem 1 from scratch using a working example. Take up again the notations preceding Theorem 1 with $\sigma$ designating a permutation of order $n$. For each $j=1, \ldots, l$ let $\tau_{j}$ be the restriction of $\sigma$ to the set $\sigma^{-1}\left(\left[\mathbf{n}_{j-1}+1, \mathbf{n}_{j}\right]\right)$, so that

$$
\tau_{j}: \sigma^{-1}\left(\left[\mathbf{n}_{j-1}+1, \mathbf{n}_{j}\right]\right) \rightarrow\left[\mathbf{n}_{j-1}+1, \mathbf{n}_{j}\right] .
$$

Using the Robinson-Schensted correspondence [Kn, p. 48-72] each bijection $\tau_{j}$ is mapped onto a pair $\left(P_{j}, Q_{j}\right)$ of Young tableaux of the same shape that we 
shall denote by $\lambda_{j}$. It follows from the properties of that correspondence that the entries of $P_{j}$ are the elements of the interval $\left[\mathbf{n}_{j-1}+1, \mathbf{n}_{j}\right]$ and the entries of $Q_{j}$ are those of the set $\sigma^{-1}\left(\left[\mathbf{n}_{j-1}+1, \mathbf{n}_{j}\right]\right)$. For $j=1, \ldots, l$ let $T_{j}$ be the Young tableau obtained from $P_{j}$ by replacing each entry $r$ by $r-\mathbf{n}_{j-1}$ and let $U$ be the product

$$
U=Q_{1} \otimes \cdots \otimes Q_{l} .
$$

In the French way of displaying tableaux this means that $U$ is the skew tableau obtained by placing each $Q_{j}$ to the right of $Q_{j-1}$ and just under it for $j=$ $2, \ldots, l$. Thus each $T_{j}$ is a Young tableau of shape $\lambda_{j}$ whose entries are $1,2, \ldots, n_{j}(j=1, \ldots, l)$; the entries of $U$ are $1,2, \ldots, n$ and the shape of $U$ is the skew shape $\lambda_{1} \otimes \cdots \otimes \lambda_{l}$. We summarize all this by writing

$$
\begin{gathered}
\text { shape } T_{j}=\lambda_{j} ; \quad\left|\lambda_{j}\right|=n_{j} ; \quad(j=1, \ldots, l) ; \\
\text { shape } U=\lambda_{1} \otimes \cdots \otimes \lambda_{l} ; \quad\left|\lambda_{1}\right|+\cdots+\left|\lambda_{l}\right|=n .
\end{gathered}
$$

For instance, let $l=2, \mathbf{n}=(5,4)$ and

$$
\begin{aligned}
\sigma & =\left(\begin{array}{lllllllll}
1 & 2 & 3 & 4 & 5 & 6 & 7 & 8 & 9 \\
8 & 6 & 4 & 5 & 2 & 1 & 9 & 7 & 3
\end{array}\right) \\
\sigma^{-1} & =\left(\begin{array}{lllll|lllll}
1 & 2 & 3 & 4 & 5 & 6 & 7 & 8 & 9 \\
6 & 5 & 9 & 3 & 4 & 2 & 8 & 1 & 7
\end{array}\right)
\end{aligned}
$$

Then

$$
\tau_{1}=\left(\begin{array}{lllll}
3 & 4 & 5 & 6 & 9 \\
4 & 5 & 2 & 1 & 3
\end{array}\right) ; \quad \tau_{2}=\left(\begin{array}{llll}
1 & 2 & 7 & 8 \\
2 & 6 & 9 & 7
\end{array}\right)
$$

Under the Robinson-Schensted correspondence

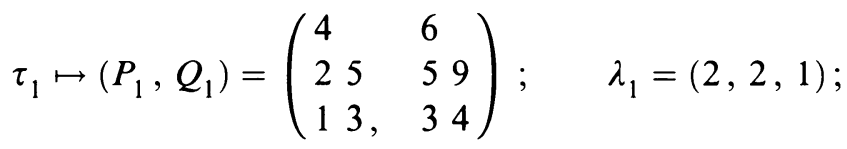

$$
\begin{aligned}
& \tau_{2} \mapsto\left(P_{2}, Q_{2}\right)=\left(\begin{array}{llll}
8 & 9 & 2 & 8 \\
6 & 7, & 1 & 7
\end{array}\right) ; \quad \lambda_{2}=(2,2) ;
\end{aligned}
$$

and

$$
\begin{array}{crr}
4 & & 6 \\
25 & 34 & 59 \\
T_{1}=P_{1}=13 ; & T_{2}=P_{2}-\mathbf{n}_{1}=12 ; \quad U=34
\end{array}
$$

The inverse ligne of route Iligne $U$ of $U$ is defined as being the set of all entries $r$ such that $(r+1)$ lies in a higher row than $r$ in $U$ (in the French way of displaying tableaux). Furthermore, define

$$
\text { imaj } U=\sum\{r: r \in \text { Iligne } U\}, \quad \operatorname{ides} U=\mid \text { Iligne } U \mid \text {. }
$$

With the working example, Iligne $U=\{1,2,4,5,7,8\}$, imaj $U=27$ and ides $U=6$. 
Lemma 2.1. The mapping $(\sigma, \mathbf{n}) \mapsto\left(\lambda_{1}, \ldots, \lambda_{l} ; T_{1}, \ldots, T_{l} ; U\right)$ is a bijection having properties (2.2) and satisfying

$$
\begin{aligned}
\text { Iligne }_{j} \sigma_{j}-\mathbf{n}_{j-1} & =\text { Iligne } T_{j} \quad(j=1, \ldots, l) ; \\
\text { Ligne } \sigma & =\text { Iligne } U .
\end{aligned}
$$

We do not prove Lemma 2.1, as it was already derived in [DeFo2] in the context of the $(k, l)$-colored permutations. With our working example Iligne ${ }_{1} \sigma=$ $\{1,3\}=$ Iligne $T_{1}$; Iligne ${ }_{2} \sigma=\{2\}=$ Iligne $T_{2}$; Ligne $\sigma=\{1,2,4,5,7,8\}=$ Iligne $U$.

It follows from (2.3) that

$$
\operatorname{imaj}_{j} \sigma=\operatorname{imaj} T_{j} \quad(j=1, \ldots, l) ; \quad \operatorname{des} \sigma=\operatorname{ides} U .
$$

Let $s^{1} A_{\mathbf{n}}(s ; \mathbf{q})$ denote the right-hand side of (1.9). Then by (2.3) and (2.4)

$$
{ }^{1} A_{\mathbf{n}}(s ; \mathbf{q})=\sum_{\left(\lambda_{j}\right)} \sum_{\left(T_{j}\right), U} s^{\text {ides } U} q_{1}^{\text {imaj } T_{1}} \cdots q_{l}^{\text {imaj } T_{l}},
$$

where the first sum is over the sequences $\left(\lambda_{1}, \ldots, \lambda_{l}\right)$ of partitions satisfying $\left|\lambda_{1}\right|=n_{1}, \ldots,\left|\lambda_{l}\right|=n_{l}$, and the second over all. sequences of tableaux $\left(T_{1}, \ldots, T_{l}, U\right)$ satisfying $(2.2)$.

The next step is to express the right-hand side of $(2.5)$ in terms of skew Schur functions. Let $S_{\theta}\left(x_{1}, x_{2}, \ldots\right)$ designate the skew Schur function associated with the skew diagram $\theta$ [Mac, p. 39]. The following lemma was proved in [DeFol].

Lemma 2.2. If $\theta$ is a skew diagram of $n$ elements, then

$$
\frac{1}{(s ; q)_{n+1}} \sum_{T} s^{\text {ides } T} q^{\text {imaj } T}=\sum_{r} s^{r} S_{\theta}\left(1, q, q^{2}, \ldots, q^{r}\right),
$$

where the first sum is over all standard tableaux $T$ of shape $\theta$.

We are using here two specializations of this lemma obtained by letting $q=1$ and $s=1$, respectively,

$$
\begin{aligned}
& \frac{1}{(1-s)^{n+1}} \sum_{T} s^{\text {ides } T}=\sum_{r} t^{r} S_{\theta}\left(1^{r+1}\right), \\
& \frac{1}{(q ; q)_{n}} \sum_{T} q^{\text {imaj } T}=S_{\theta}\left(1, q, q^{2}, \ldots\right),
\end{aligned}
$$

where $S_{\theta}\left(1^{r+1}\right)$ is the skew Schur function obtained by taking an alphabet of $(r+1)$ letters all equal to 1 . 
By (2.5), (2.6) and (2.7) we have

$$
\begin{aligned}
\frac{{ }^{1} A_{\mathbf{n}}(s, \mathbf{q})}{(1-s)^{n} \prod_{j}\left(q_{j} ; q_{j}\right)_{n_{j}}} & =\frac{1}{(1-s)^{n} \prod_{j}\left(q_{j} ; q_{j}\right)_{n_{j}}} \sum_{\left(\lambda_{j}\right),\left(T_{j}\right), U} s^{\operatorname{ides} U} \prod_{j} q_{j}^{\text {imaj } T_{j}} \\
& =\sum_{\left(\lambda_{j}\right)} \sum_{r} s^{r} S_{\lambda_{1} \otimes \cdots \otimes \lambda_{l}}\left(1^{r+1}\right) \prod_{j} S_{\lambda_{j}}\left(1, q_{j}, q_{j}^{2}, \ldots\right) \\
& =\sum_{\left(\lambda_{j}\right)} \sum_{r} s^{r} \prod_{j} S_{\lambda_{j}}\left(1^{r+1}\right) S_{\lambda_{j}}\left(1, q_{j}, q_{j}^{2}, \ldots\right),
\end{aligned}
$$

as $U$ is of shape $\lambda_{1} \otimes \cdots \otimes \lambda_{l}$. In the last step we have used the fundamental multiplicative property of the Schur functions: $S_{\lambda \otimes \mu}(x)=S_{\lambda}(x) S_{\mu}(x)$. Now the Cauchy identity for Schur functions

$$
\sum_{\lambda} u^{|\lambda|} S_{\lambda}(x) S_{\lambda}(y)=\prod_{i, j} \frac{1}{\left(1-u x_{i} y_{i}\right)}
$$

yields

$$
\begin{aligned}
& \sum_{\lambda_{j}} u_{j}^{\left|\lambda_{j}\right|} S_{\lambda_{j}}\left(1^{r+1}\right) S_{\lambda_{j}}\left(1, q_{j}, q_{j}^{2}, \ldots\right) \\
& \quad=\prod_{d \geq 1} \frac{1}{\left(1-u_{j} q_{j}^{d-1}\right)^{r+1}}=\frac{1}{\left(u_{j} ; q_{j}\right)_{\infty}^{r+1}} .
\end{aligned}
$$

As $\left|\lambda_{j}\right|=n_{j}(j=1, \ldots, l)$, we may write

$$
\begin{aligned}
\sum_{\mathbf{n}} & \frac{\prod_{j} u_{j}^{n_{j}}}{(1-s)^{n} \prod_{j}\left(q_{j} ; q_{j}\right)_{n_{j}}} A_{\mathbf{n}}(s, \mathbf{q}) \\
& =\sum_{\mathbf{n}} \prod_{j} u_{j}^{n_{j}} \sum_{\left(\lambda_{j}\right)} \sum_{r} s^{r} \prod_{j} S_{\lambda_{j}}\left(1^{r+1}\right) S_{\lambda_{j}}\left(1, q_{j}, q_{j}^{2}, \ldots\right) \\
& =\sum_{r} s^{r} \sum_{\left(\lambda_{j}\right)} \prod_{j} u_{j}^{\left|\lambda_{j}\right|} S_{\lambda_{j}}\left(1^{r+1}\right) S_{\lambda_{j}}\left(1, q_{j}, q_{j}^{2}, \ldots\right) \\
& =\sum_{r} s^{r} \prod_{j} \sum_{\lambda_{j}} u_{j}^{\left|\lambda_{j}\right|} S_{\lambda_{j}}\left(1^{r+1}\right) S_{\lambda_{j}}\left(1, q_{j}, q_{j}^{2}, \ldots\right) \\
& =\sum_{r} \prod_{j} \frac{s^{r}}{\left(u_{j} ; q_{j}\right)_{\infty}^{r+1}}=\frac{1}{-s+\prod_{j}\left(u_{j} ; q_{j}\right)_{\infty}} .
\end{aligned}
$$

Next replace each $u_{j}$ by $u_{j} /(1-s)$. This yields

$$
\begin{aligned}
\sum_{\mathbf{n}} \frac{\prod_{j} u_{j}^{n_{j}}}{\prod_{j}\left(q_{j} ; q_{j}\right)_{n_{j}}}{ }^{1} A_{\mathbf{n}}(s, \mathbf{q}) & =\frac{1-s}{-s+\prod_{j}\left((1-s) u_{j} ; q_{j}\right)_{\infty}} \\
& =\frac{1-s}{-s+\prod_{j} E\left((s-1) u_{j} ; q_{j}\right)},
\end{aligned}
$$

using the notation of the second $q$-exponential introduced in (1.3). 
The generating function for the polynomials $A_{\mathbf{n}}(s, \mathbf{q})$ involves the first $q$ exponential, as shown in the next calculation

$$
\begin{aligned}
1+\sum_{\mathbf{n} \neq 0} \frac{\prod_{j} u_{j}^{n_{j}}}{\prod_{j}\left(q_{j} ; q_{j}\right)_{n_{j}}} A_{\mathbf{n}}(s, \mathbf{q}) & =s \frac{1-s}{-s+\prod_{j} E\left((s-1) u_{j} ; q_{j}\right)}-s+1 \\
& =\frac{1-s}{1-s \prod_{j} e\left((1-s) u_{j} ; q_{j}\right)},
\end{aligned}
$$

since $E(u ; q) e(-u ; q)=1$. This proves Theorem 1 .

\section{Proof of Theorem 2}

Call $A_{\mathbf{n}}^{\text {inv }}(s ; \mathbf{q})$ the right-hand side of $(1.10)$, namely the generating function for $\mathscr{S}_{n}$ by the vector $\left(1+\operatorname{des}, \operatorname{inv}_{1}, \ldots\right.$, inv $\left._{l}\right)$. We will prove that $A_{\mathbf{n}}^{\text {inv }}(s ; \mathbf{q})$ satisfies recurrence (1.5), i.e., the equation obtained from (1.5) by replacing $A_{\mathrm{n}}$ and $A_{\mathbf{i}}$ by $A_{\mathrm{n}}^{\text {inv }}$ and $A_{\mathbf{i}}^{\text {inv }}$, respectively. Since $A_{0}=A_{\mathbf{0}}^{\text {inv }}=1$ by convention, the result will follow by induction.

Consider the set $\mathscr{T}_{n}$ of all "commaed" permutations that consist of a permutation in $\mathscr{S}_{n}$ together with a comma inserted in such a way that the sequence after the comma is increasing. For example, 4713, 256; 47132, 56; 471325,6 ; are all members of $\mathscr{T}_{n}$, but 47,13256 is not.

To each member $\left(\sigma_{1}, \sigma_{2}\right)$ (i.e., $\sigma_{2}$ is increasing and nonempty and $\sigma:=\sigma_{1} \sigma_{2}$ is a regular permutation of $\mathscr{S}_{n}$ ) of $\mathscr{T}_{n}$ associate the weight

$$
\text { weight }\left(\sigma_{1}, \sigma_{2}\right)=s^{1+\operatorname{des} \sigma_{1}} s(1-s)^{\text {length } \sigma_{2}-1} \prod_{j} q_{j}^{\text {inv }}{ }^{\text {o }} .
$$

Next to each $\sigma$ in $\mathscr{S}_{n}$ associate the set $C(\sigma)$ of all commaed permutations obtained from $\sigma$ by inserting a comma right before one of the entries that belong to the maximal terminal increasing right factor of $\sigma$.

For example, $\sigma=4713256$ gives rise to the following set

$$
C(\sigma)=\{4713,256 ; 47132,56 ; 471325,6\} .
$$

We claim that this mapping $C$ is weight preserving, i.e.,

$$
s^{1+\operatorname{des} \sigma} \prod_{j} q_{j}^{\text {inv }_{j} \sigma}=\sum_{\tau \in C(\sigma)} \text { weight } \tau .
$$

Indeed the $\prod_{j} q_{j}^{\text {inv }_{j} \sigma}$ factor is the same at both sides. The $s$-contribution is as follows, letting $r$ be the length of the maximal increasing right factor of $\sigma$,

$$
\begin{aligned}
& \operatorname{weight}(\sigma(1) \cdots \sigma(n-r))\left[s(1-s)^{r-1}+s^{2}(1-s)^{r-2}+\cdots+s^{2}(1-s)^{0}\right] \\
& \quad=\operatorname{weight}(\sigma(1) \cdots \sigma(n-r)) s=\operatorname{weight}(\sigma(1) \cdots \sigma(n))=\operatorname{weight}(\sigma) .
\end{aligned}
$$

Thus the sum of the weights of all the elements of $\mathscr{S}_{n}\left(\right.$ i.e., $\left.A_{\mathbf{n}}^{\text {inv }}(s ; \mathbf{q})\right)$ is the same as the sum of all the weights of the elements of $\mathscr{T}_{n}$. Let us compute the latter, noting that $\operatorname{inv}_{j} \sigma$ is also equal to

$$
\operatorname{inv}_{j} \sigma=\#\left\{\left(r, r^{\prime}\right): 1 \leq r<r^{\prime} \leq n, \mathbf{n}_{j} \geq \sigma(r)>\sigma\left(r^{\prime}\right) \geq \mathbf{n}_{j-1}+1\right\} .
$$


Consider the subset of $\mathscr{T}_{n}$ consisting of all elements such that the comma comes right after $i_{1}$ members of the first class, $\ldots, i_{l}$ members of the $l$ th class. Let $h_{1}=n_{1}-i_{1}, \ldots, h_{l}=n_{l}-i_{l}$ and assume that after the comma we have the fixed increasing factor $a_{1}^{(1)} \cdots a_{h_{1}}^{(1)} \cdots a_{1}^{(l)} \cdots a_{h_{l}}^{(l)}$, with $\mathbf{n}_{j-1}+1 \leq a_{1}^{(j)}<\cdots<a_{h_{j}}^{(j)} \leq \mathbf{n}_{l}$ for $j=1, \ldots, l$. The total weight of the above factor is easily seen to be

$$
\begin{aligned}
& A_{\mathbf{i}}^{\text {inv }}(s ; \mathbf{q}) s(1-s)^{n_{1}-i_{1}+n_{2}-i_{2}+\cdots+n_{l}-i_{l}-1} \\
& \quad \times \prod_{j} q_{j}^{\left(n_{j}-a_{h_{j}}^{(j)}\right)+\left(n_{j}-a_{h_{j}-1}^{(j)}-1\right)+\cdots+\left(n_{j}-a_{j}^{(j)}-\left(h_{j}-1\right)\right)} .
\end{aligned}
$$

Now for a fixed $\mathbf{i}=\left(i_{1}, i_{2}, \ldots, i_{l}\right)$ we have that the sum runs independently with respect to each of the exponents of the $q_{j}$ 's. It is easy to see that inside the exponent of $q_{j} \quad(j=1, \ldots, l)$ we have a typical partition of $n$ with largest part at most equal to $n_{j}-h_{j}\left(=i_{j}\right)$ and number of parts at most equal to $h_{j}$. The generating function of which is well known to be the $q$-binomial $\left[\begin{array}{l}n_{j} \\ i_{j}\end{array}\right]_{q_{j}}$ to base $q_{j}$. Since the sums over each $q_{j}$ run independently, the total weight of members of $\mathscr{T}_{n}$ such that right before the comma come $i_{1}$ members of the first class, $\ldots, i_{l}$ members of the $l$ th class (regardless of what comes after) is

$$
\prod_{j}\left[\begin{array}{c}
n_{j} \\
i_{j}
\end{array}\right]_{q_{j}} A_{\mathbf{i}}^{\text {inv }}(s ; \mathbf{q}) s(1-s)^{n_{1}-i_{1}+\cdots+n_{l}-i_{l}-1} .
$$

Summing over all conceivable $\mathbf{i} \neq \mathbf{n}$ establishes (1.5).

The technique used here was inspired by some methods developed in [Ge2] and $[\mathrm{Ze}]$.

\section{INVERSION NUMBERS}

The proof of Theorem 3 relies upon the properties of the so-called second fundamental transformation [Lot, Chapter 10], which we shall designate by $\phi$. Let $w=x_{1} \cdots x_{m}$ be a word whose letters belong to $\mathbf{N}$ and let $x$ be an integer. If the last letter $w$ is greater (resp. smaller than or equal to) $x$, the word $w$ admits a unique factorization $\left(w_{1} y_{1}, \ldots, w_{p} y_{p}\right)$, called its $x$-factorization having the following properties:

(i) each $y_{i}(i=1, \ldots, p)$ satisfies $y_{i}>x$ (resp. $\left.y_{i} \leq x\right)$;

(ii) each word $w_{i}(i=1, \ldots, p)$ is either empty, or has all its letters smaller than or equal to (resp. greater than) $x$.

Define $\gamma_{x}(w)=y_{1} w_{1} \cdots y_{p} w_{p}$. (Note that $w=w_{1} y_{1} \cdots w_{p} y_{p}$. ) The transformation $\phi$ is defined by induction as follows: $\phi(w)=w$, if $w$ is of length one; if $w$ has a length at least equal to 2 , write $w=v x$ with $x$ being the last letter of $w$, and define $\phi(v x)=\gamma_{x}(\phi(v)) x$. Let $W$ be the rearrangement class of some word $w_{0}$. It was proved in [Fo] that $\phi$ is a bijection of $W$ onto itself having the property that $\operatorname{maj} w=\operatorname{inv} \phi(w)$,

for every $w \in W$. 
A further property of $\phi$ was stated and proved in [FoSch] for the rearrangement classes $W$ containing only words without repetitions, say, subwords of permutations. If $w$ is such a word, its inverse ligne of route Iligne $w$ may be defined as being the set of all $k$ such that $k$ and $(k+1)$ are letters of $w$ and $(k+1)$ is to the left of $k$ in $w$. In particular, if $w$ is a permutation of $1,2, \ldots, n$, then Iligne $w$ is simply the ligne of route of the inverse permutation $w^{-1}$. Thus when the rearrangement class $W$ contains only subwords of permutations, it was shown in [FoSch] that

$$
\text { Iligne } w=\text { Iligne } \phi(w) \text {. }
$$

Recently, Björner and Wachs [BjWa] have extended the properties of the second fundamental transformation to a larger set of combinatorial objects, and found several characterizations for sets that satisfied (4.2). Our purpose presently is to include $\phi$ in the construction of a bijection that will serve to prove Theorem 3.

If $\sigma=\sigma(1) \cdots \sigma(n)$ is a permutation and if $\mathbf{n}=\left(n_{1}, \ldots, n_{l}\right)$ is a sequence of integers of sum $n$, let

$$
\sigma_{j}=\sigma\left(\mathbf{n}_{j-1}+1\right) \sigma\left(\mathbf{n}_{j-1}+2\right) \cdots \sigma\left(\mathbf{n}_{j}+1\right)
$$

for $j=1, \ldots, l$. If $\Theta$ is any transformation on $\mathscr{S}_{n}$, we will write

$$
(\Theta \sigma)_{j}=\Theta \sigma\left(\mathbf{n}_{j-1}+1\right) \Theta \sigma\left(\mathbf{n}_{j-1}+2\right) \cdots \Theta \sigma\left(\mathbf{n}_{j}+1\right) .
$$

In particular, if we apply the second fundamental transformation $\phi$ to each $\sigma_{j}$ and form the juxtaposition product

$$
\psi(\sigma)=\phi\left(\sigma_{1}\right) \cdots \phi\left(\sigma_{l}\right),
$$

we define a new transformation on $\mathscr{S}_{n}$. Furthermore, $\phi\left(\sigma_{j}\right)=(\psi \sigma)_{j}$ using the previous notations.

Proposition 4.1. The sequence $\mathbf{n}$ being given, the mapping $\psi$ is a bijection of $\mathscr{S}_{n}$ onto itself satisfying

$$
\operatorname{maj} \sigma_{j}=\operatorname{inv}(\psi \sigma)_{j}, \quad(j=1, \ldots, l)
$$

Iligne $\sigma=$ Iligne $\psi(\sigma)$.

Proof. Relation (4.4) is just (4.1) rewritten for the factors $\sigma_{j}$. Now by (4.2) we have Iligne $\sigma_{j}=$ Iligne $\phi\left(\sigma_{j}\right)$. This takes care of all the pairs $(k, k+1)$ contained in a single factor $\sigma_{j}$, and therefore in a factor $\phi\left(\sigma_{j}\right)$. Next, if $1 \leq i<j \leq l$ and $(k+1)$ is a letter of $\sigma_{i}$, while $k$ is a letter of $\sigma_{j}$, then $(k+1)$ will be to the left of $k$ both in $\sigma$ and $\psi(\sigma)$.

Let $\mathbf{i} \sigma=\sigma^{-1}$ and form the sequence

$$
\sigma \mapsto \mathbf{i} \sigma \mapsto \psi \mathbf{i} \sigma \mapsto \mathbf{i} \psi \mathbf{i} \sigma,
$$

By (4.5)

$$
\text { Ligne } \sigma=\text { Iligne } \mathbf{i} \sigma=\text { Iligne } \psi \mathbf{i} \sigma=\operatorname{Ligne} \mathbf{i} \psi \mathbf{i} \sigma
$$


Furthermore, by (4.4)

$$
\operatorname{maj}(\mathbf{i} \sigma)_{j}=\operatorname{inv}(\psi \mathbf{i} \sigma)_{j} \quad(j=1, \ldots, l) .
$$

Define

$$
\sigma^{\prime}:=\mathbf{i} \psi \mathbf{i} \sigma
$$

Then $\sigma \mapsto \sigma^{\prime}$ is a bijection satisfying Ligne $\sigma=$ Ligne $\sigma^{\prime}$ by (4.6). Furthermore,

$$
\operatorname{imaj}_{j} \sigma_{j}=\operatorname{maj}(\mathbf{i} \sigma)_{j}=\operatorname{inv}(\psi \mathbf{i} \sigma)_{j}=\operatorname{inv}\left(\sigma^{\prime-1}\right)_{j}=\operatorname{inv}_{j} \sigma^{\prime} .
$$

This completes the proof of Theorem 3.

\section{The DÉSARMÉNIEN Verfahren}

Let $x=\left(x_{1}, x_{2}, \ldots\right)$ be an infinite sequence of variables and for each $r=$ $1,2, \ldots$ denote by $h_{r}(x)$ the homogeneous symmetric function in the $x_{j}$ 's and by $p_{r}(x)$ the power sum $\sum_{j} x_{j}^{r}$. By convention, $h_{0}(x)=1$. The generating function $H(u ; x)=\sum_{r \geq 0} u^{r} h_{r}(x)$ can be evaluated in different forms:

$$
H(u ; x)=\prod_{j \geq 1}\left(1-u x_{j}\right)^{-1}=\exp \sum_{r \geq 1} u^{r} \frac{p_{r}(x)}{r} .
$$

(See, e.g., [Mac, pp. 14 and 17].)

Recall that a partition of an integer $n$ can be expressed as a nonincreasing sequence $\lambda=\left(\lambda_{1}, \lambda_{2}, \ldots\right)\left(\lambda_{1} \geq \lambda_{2} \geq \cdots\right)$, or as a word $\lambda=1^{m_{1}} 2^{m_{2}} \ldots$ (the multiplicative notation) with the meaning that $\lambda$ has $m_{1}$ parts $\lambda_{i}$ equal to 1 , $m_{2}$ parts $\lambda_{i}$ equal to 2 , etc... As usual, to each partition $\lambda$ we attach the constant

$$
z_{\lambda}=1^{m_{1}} 2^{m_{2}} \cdots m_{1} ! m_{2} ! \cdots
$$

and the power symmetric function

$$
p_{\lambda}(x)=p_{\lambda_{1}}(x) p_{\lambda_{2}}(x) \cdots
$$

Also $|\lambda|=n$ means that $\lambda$ is a partition of $n$ and the notation $l(\lambda)$ stands for the number of parts of $\lambda$.

Désarménien [De] introduced the polynomials

$$
T_{\lambda}(q)=\frac{(q ; q)_{n}}{\prod_{j}\left(1-q^{j}\right)^{m_{j}}},
$$

where $\lambda=1^{m_{1}} 2^{m_{2}} \ldots$ is a partition of $n$. He noticed that $T_{\lambda}(q)$ is a polynomial of degree $n(n-1) / 2$ and can also be expressed as

$$
T_{\lambda}(q)=(q ; q)_{n} p_{\lambda}\left(1, q, q^{2}, \ldots\right) .
$$

Furthermore, he proved the following congruence property. 
Lemma 5.1. Let $n=d a+b, 0 \leq b \leq d-1$ and $\lambda=1^{m_{1}} 2^{m_{2}} \ldots$ be a partition of $n$. Then the following congruences hold:

(i) if $m_{d} \neq a$, then $T_{\lambda}(q) \equiv 0\left(\bmod \Phi_{d}(q)\right)$.

(ii) if $m_{d}=a$, let $\mu=\lambda \backslash d^{a}$ be the partition obtained from $\lambda$ by deleting the $m_{d}$ parts equal to $d$. Then

$$
T_{\lambda} \equiv d^{a} a ! T_{\mu}(q) \quad\left(\bmod \Phi_{d}(q)\right) .
$$

Now let $\left(c_{i}\right)(i=0,1, \ldots)$ be a sequence of elements belonging to some given ring. The relation

$$
\sum_{i \geq 0} C_{i} \frac{u^{i}}{i !}=\sum_{i \geq 0} c_{i}\left(e^{u}-1\right)^{i}
$$

defines a sequence $\left(C_{i}\right)$ in a unique manner.

Désarménien considered the expansion

$$
\sum_{m \geq 0, n \geq 0} \mathscr{C}_{n, m}(x) u^{n} \frac{v^{m}}{m !}=\sum_{i \geq 0} c_{i}\left(H(u ; x) e^{v}-1\right)^{i}
$$

and showed that $\mathscr{C}_{n, m}(x)$ can be expressed in terms of the symmetric functions $\left(p_{\lambda}(x)\right)$ as

$$
\mathscr{C}_{n, m}(x)=\sum_{\lambda} C_{m+l(\lambda)} \frac{p_{\lambda}(x)}{z_{\lambda}} \quad(|\lambda|=n) .
$$

Then he defined the polynomials

$$
K_{m, n}(q)=(q ; q)_{n} \mathscr{C}_{m, n}\left(1, q, q^{2}, \ldots\right),
$$

and using Lemma 5.1 proved the congruence

$$
K_{m, d a+b}(q) \equiv K_{m+a, b}(q) \quad\left(\bmod \Phi_{d}(q)\right) .
$$

Finally, he applied the latter congruence to his $q$-Eulerian polynomials with two indices to obtain Theorem 4 for $k=1$.

The program for an arbitrary $k$ and consequently for the multibasic Eulerian polynomials will closely follow the foregoing pattern. This time we have to introduce $l$ sequences of variables $x^{j}=\left(x_{1}^{j}, x_{2}^{j}, \ldots\right)(1 \leq j \leq l)$, and relation (5.4) being given we expand the expression $\sum_{i \geq 0} c_{i}\left(\prod_{j} H\left(u_{j} ; x^{j}\right)-1\right)^{i}$, as a power series in the $u_{j}$ 's. This is done as follows.

Proposition 5.2. If

$$
\left.\sum_{\mathbf{n}} \prod_{j=1}^{l} u_{j}^{n_{j}} \mathscr{C}_{n}=\sum_{i \geq 0} c_{i}\left(\prod_{j=1}^{l} H\left(u_{j} ; x^{j}\right)-1\right)\right)^{i},
$$

then

$$
\mathscr{C}_{\mathbf{n}}=\sum_{\lambda^{\prime}, \ldots, \lambda^{\prime}} C_{l\left(\lambda^{1}\right)+\cdots+l\left(\lambda^{\prime}\right)} \prod_{j=1}^{l} \frac{p_{\lambda^{j}}\left(x^{j}\right)}{z_{\lambda^{j}}},
$$

the sum being over all partitions satisfying $\left|\lambda^{1}\right|=n_{1}, \ldots,\left|\lambda^{l}\right|=n_{l}$. 
The proof has been made in [DeFo2] in the case $l=2$ and $H\left(u_{2} ; x^{2}\right)=e^{u_{2}}$. The proof for an arbitrary $l$ is quite similar and will therefore be left out.

Let $0 \leq k$ and $k+1=l$ and suppose that $H\left(u_{l} ; x^{l}\right)$ is equal to $e^{u_{l}}$. From (5.1) it follows that $p_{1}\left(x^{l}\right)=1$ and $p_{r}\left(x^{l}\right)=0$ for $r \geq 2$. Hence, if $\left|\lambda^{l}\right|=n_{l}$, we have

$$
\frac{p_{\lambda^{l}}\left(x^{l}\right)}{z_{\lambda^{l}}}= \begin{cases}0, & \text { if } \lambda^{l} \neq\left(1^{n_{l}}\right) \\ 1 / n_{l} !, & \text { if } \lambda^{l}=\left(1^{n_{l}}\right)\end{cases}
$$

Hence (5.6) takes the form

$$
\mathscr{C}_{\mathbf{n}} \sum_{\lambda^{1}, \ldots, \lambda^{k}} C_{l\left(\lambda^{1}\right)+\cdots+l\left(\lambda^{k}\right)+n_{k+1}} \prod_{j=1}^{k} \frac{p_{\lambda^{j}}\left(x^{j}\right)}{z_{\lambda^{j}}} \frac{1}{n_{k+1} !},
$$

where $\left|\lambda^{1}\right|=n_{1}, \ldots,\left|\lambda^{k}\right|=n_{k}$, and (5.5) the form

$$
\sum_{\mathbf{n}} \prod_{j=1}^{k+1} u_{j}^{n_{j}} \mathscr{C}_{n}=\sum_{i \geq 0} c_{i}\left(\prod_{j=1}^{k} H\left(u_{j} ; x^{j}\right) e^{u_{k+1}}-1\right)^{i} \text {. }
$$

For $j=1, \ldots, k$ and $r=1,2, \ldots$ replace each $x_{r}^{j}$ by $q_{j}^{r-1}$ in (5.8) and (5.9). This substitution being made, define

$$
K_{\mathbf{n}}\left(q_{1}, \ldots, q_{k}\right)=\left.\prod_{j=1}^{k}\left(q_{j} ; q_{j}\right)_{n_{j}} n_{k+1} ! \mathscr{C}_{n}\right|_{x_{r}^{j} \leftarrow q_{j}^{r-1}} .
$$

By (5.3) and (5.8) we obtain

$$
K_{\mathbf{n}}\left(q_{1}, \ldots, q_{k}\right)=\sum_{\left|\lambda^{1}\right|=n_{1}, \ldots,\left|\lambda^{k}\right|=n_{k}} \mathscr{C}_{l\left(\lambda^{1}\right)+\cdots+l\left(\lambda^{k}\right)+n_{k+1}} \prod_{j=1}^{k} \frac{T_{\lambda^{j}}\left(q_{j}\right)}{z_{\lambda^{j}}} .
$$

On the other hand, by (1.2) and (5.1)

$$
\left.H\left(u_{j} ; x^{j}\right)\right|_{x_{r}^{j} \leftarrow q_{j}^{r-1}}=\prod_{r \geq 1}\left(1-u_{j} q_{j}^{r-1}\right)^{-1}=e\left(u_{j} ; q_{j}\right) .
$$

Hence (5.9) becomes

$$
\sum_{\mathbf{n}}=\frac{\prod_{j=1}^{l+1} u_{j}^{n_{j}}}{\prod_{j=1}^{k}\left(q_{j} ; q_{j}\right)_{n_{j}} n_{k+1} !} K_{\mathbf{n}}\left(q_{1}, \ldots, q_{k}\right)=\sum_{i \geq 0}\left(\prod_{j=1}^{k} e\left(u_{j} ; q_{j}\right) e^{u_{k+1}}-1\right)^{i} .
$$

Remark. In the notations of Lemma 5.1, case (ii) $\left(m_{d}=a\right)$ we have $l(\lambda)=$ $l(\mu)+a$ and $z_{\lambda}=z_{\mu} d^{a} a !$. Hence

$$
\frac{T_{\lambda}(q)}{z_{\lambda}} \equiv \frac{T_{\mu}(q)}{z_{\mu}} \quad\left(\bmod \Phi_{d}(q)\right) .
$$


Now let $\left(d_{1}, \ldots, d_{k}\right)$ be given and let $n_{j}=d_{j} a_{j}+b_{j} \quad\left(0 \leq b_{j} \leq d_{j}-1\right.$; $1 \leq j \leq k)$. When $K_{\mathrm{n}}$ is taken $\bmod \left(\Phi_{d_{1}}\left(q_{1}\right), \ldots, \Phi_{d_{k}}\left(q_{k}\right)\right)$, formula $(5.10)$ becomes in view of Lemma 5.1 and (5.12):

$$
K_{\mathbf{n}}(\mathbf{q})=\sum_{\left|\mu^{1}\right|=b_{1}, \ldots,\left|\mu^{k}\right|=b_{k}} \mathscr{C}_{l\left(\mu^{1}\right)+a_{1}+\cdots+l\left(\mu^{k}\right)+a_{k}+n_{k+1}} \prod_{j=1}^{k} \frac{T_{\mu^{j}}\left(q_{j}\right)}{z_{\mu^{j}}} .
$$

But by (5.10) the right-hand side of the previous congruence is also equal to $K_{\text {div } \mathbf{n}}(\mathbf{q})$ with

$$
\operatorname{div} \mathbf{n}=\left(b_{1}, \ldots, b_{k}, n_{k+1}+a_{1}+\cdots+a_{k}\right) .
$$

Hence

$$
K_{\mathbf{n}}(\mathbf{q}) \equiv K_{\operatorname{div} \mathbf{n}}(\mathbf{q}) \quad\left(\bmod \left(\Phi_{d_{1}}\left(q_{1}\right), \ldots, \Phi_{d_{k}}\left(q_{k}\right)\right)\right) .
$$

To apply the foregoing congruence results to the multibasic Eulerian polynomials we note that (1.6) can be rewritten as

$$
\begin{aligned}
& \sum_{\mathbf{n}} \frac{\prod_{j=1}^{k+1} u_{j}^{n_{j}}}{\prod_{j=1}^{k}\left(q_{j} ; q_{j}\right)_{n_{j}} n_{k+1} !} \frac{B_{\mathbf{n}}(s ; \mathbf{q})}{(1-s)^{n_{1}+\cdots+n_{k+1}}} \\
& \quad=\sum_{i \geq 0}\left(\frac{s}{1-s}\right)^{i}\left(\prod_{j=1}^{k} e\left(u_{j} ; q_{j}\right) e^{u_{k+1}}-1\right)^{i} .
\end{aligned}
$$

Comparing the latter identity with (5.11) we see that we can apply (5.13) to $K_{\mathbf{n}}(\mathbf{q})=B_{\mathbf{n}}(s ; \mathbf{q}) /(1-s)^{n_{1}+\cdots+n_{k+1}}$, which are polynomials in the $q_{j}$ 's with coefficients in the field $\mathbf{Q}(s)$. This gives immediately Theorem 4 .

Consider a polynomial $B_{\mathbf{n}}(s ; \mathbf{q}) \quad\left(\mathbf{n} \in \mathbf{N}^{k+1}\right)$ and suppose that the first $k$ components of $\mathbf{n}$ are equal to 0 or 1 . It then follows from (1.7) and Theorem 1 that there is no variable $q_{j}$ occurring in the expression of $B_{\mathbf{n}}(s ; \mathbf{q})$. Therefore, this polynomial reduces to the ordinary Eulerian polynomial $A_{n_{1}+\cdots+n_{k+1}}(s)$ defined in (1.1). The corollary is then a consequence of this observation and Theorem 4 .

\section{A WACHS-STYLE PROOF OF THEOREM 4}

We will give a Wachs-style proof of Theorem 2 of [DeFo2], i.e., the onecolored case of Theorem 4 . The same argument then goes to prove Theorem 4 itself. We use the combinatorial interpretation of Theorem 2.

We have to prove that if $\omega=$ primitive $d$ th root of unity, then

$$
B_{d a+b, m}(s ; \omega)=(1-s)^{(d-1) a} B_{b, m+a}(s, \omega) .
$$

Let us first prove the case $a=1, b=0, m=0$, i.e., that $B_{d, 0}(s ; \omega)=$ $(1-s)^{d-1} B_{0,1}(s ; \omega)=(1-s)^{d-1} A_{1}(s)=(1-s)^{d-1} s$. This follows from recurrence (1.5) with one color, and the fact that

$$
\left[\begin{array}{c}
d \\
i
\end{array}\right]_{q}=\frac{\left(1-q^{d}\right)\left(1-q^{d-1}\right) \cdots\left(1-q^{d-i+1}\right)}{\left(1-q^{i}\right) \cdots(1-q)}
$$


vanishes when $q=\omega$ and $1 \leq i<d$. Thus by plugging in $q=\omega$ in (1.5) there is only one surviving term, $i=0$. We summarize this result as

$$
\sum_{\mathscr{S}_{d+0}} \text { weight } \sigma=(1-s)^{d-1} s .
$$

Now let us prove $(6.1)$ in general. We may write

$$
B_{d a+b, m}(s ; q)=\sum_{\mathscr{S}_{(d a+b)+m}} \text { weight } \pi=\sum_{\pi} s^{1+\operatorname{des} \pi} q^{\text {inv }_{1} \pi},
$$

with $\pi$ running over $\mathscr{S}_{(d a+b)+m}$, so that only the elements $1,2, \ldots, d a+b$ are taken into account for the calculation of the exponent of $q$. Group those $(d a+b)$ colored elements into families of consecutive $d$ elements

$V_{1}:=\{1, \ldots, d\} ; V_{2}:=\{d+1, \ldots, 2 d\} ; \ldots ; V_{a}:=\{(a-1) d+1, \ldots, a d\} ;$

plus a set of "bachelors"

$$
W:=\{a d+1, \ldots, a d+b\} .
$$

It is possible to view a permutation as a "seating" in a one-row auditorium. From now on "seating" and "permutation" will be used interchangeably. A happy seating is one in which no family had to split, i.e., every family sits in $d$ consecutive chairs. An unhappy seating is one in which at least one of the $d$ families had to split. We claim

(i) The sum of all the weights of the happy seatings is

$$
(1-s)^{(d-1) a} B_{b, m+a}(s ; \omega) \text {. }
$$

(ii) The sum of the weights of all the unhappy seatings is zero.

Proof of $(\mathrm{i})$. Each happy seating can be characterized by a sequence $\pi=\left(\sigma, \pi_{1}\right.$, $\left.\ldots, \pi_{a}\right)$, where $\sigma$ belongs to $\mathscr{S}_{(b)+(m+a)}$ and indicates how the $b$ bachelors, the $m$ uncolored elements and the $a$ family-blocks are displayed on the onerow seating and where $\pi_{1}, \ldots, \pi_{a}$ are permutations that arrange the seatings within each family. We have

$$
\operatorname{des} \pi=\operatorname{des} \sigma+\operatorname{des} \pi_{1}+\cdots+\operatorname{des} \pi_{a},
$$

since the total number of descents is the sum of the inter-family and intra-family descents. We also have that the number of inversions of $\pi$ (that count, i.e., the inversions between $d a+b$ elements themselves, disregarding relationships with the $m$ elements that "do not count") equals $d^{2}$ times the number of inversions in $\sigma$ between the $a$ family blocks, plus $d$ times the number of inversions that result between the $b$ bachelors and the $a$ family blocks. To these we have to add, of course, the "intra-family" inversions, i.e., inv $\pi_{1}+\cdots+$ inv $\pi_{a}$, and finally we have to add the inversions between the $b$ bachelors. Moding out by $d$ the contributions from the first two kinds of inversions vanish and we have (recall $q=\omega, \omega$ a primitive $d$ th root of unity)

$$
\text { weight } \pi=s^{1+\operatorname{des} \sigma} q^{\operatorname{inv}_{1} \sigma_{s}}{ }^{\operatorname{des} \pi_{1}} q^{\operatorname{inv} \pi_{1}} \cdots s^{\operatorname{des} \pi_{a}} q^{\operatorname{inv} \pi_{a}},
$$


so that

$$
s^{a} \text { weight } \pi=\text { weight } \sigma \text { weight } \pi_{1} \cdots \text { weight } \pi_{a} .
$$

Thus

$$
\begin{aligned}
\sum_{\pi \text { happy }} \text { weight } \pi & =s^{-a} \sum_{S_{b+(m+a)}} \text { weight } \sigma \sum_{s_{d}} \text { weight } \pi_{1} \cdots \sum_{s_{d}} \text { weight } \pi_{a} \\
& =B_{b, m+a}(s ; \omega)(1-s)^{(d-1) a},
\end{aligned}
$$

by (6.2) and (6.3).

Proof of (ii). For any set $X=\left\{x_{1}<x_{2}<\cdots<x_{L}\right\}$ and any permutation $\rho=x_{i_{1}} x_{i_{2}} \cdots x_{i_{L}}$ of $X$ we denote by red $\rho$ the permutation of $1,2, \ldots, L$, called the reduction of $\rho$, defined by $\operatorname{red} \rho=i_{1} i_{2} \cdots i_{L}$.

Michele Wachs $(d=2)$ killed bad guys in pairs. We would have to exterminate them in bunches. Let $r \quad(1 \leq r \leq a)$ be the smallest unhappy family (i.e., $r$ is the smallest integer for which $V_{r}$ is an unhappy family). Let the connected components of the seats occupied by the $V_{r}$-family members have lengths $\alpha_{1}, \ldots, \alpha_{c}$. Obviously $c \geq 2$ and $\alpha_{1}+\alpha_{2}+\cdots+\alpha_{c}=d$.

Consider all permutations that give rise to the same unhappy $r$, same component sizes $\alpha_{1}, \ldots, \alpha_{c}$, whose last connected component reduces to the same permutation $p \in \mathscr{S}_{\alpha_{c}}$ and in which all the non- $V_{r}$ objects stay the same. In other words, once the show has begun, only the $V_{r}$-family members can move seats between themselves, but they must observe the same "pattern" in the last component. We will now show that the sum of all the weights of the seatings that are related in such a way is zero.

The descents and inversions with "the outside world" remains fixed, as do the descents and inversions within "the outside world." All the changes are the descents and inversions due to the placements of the $V_{r}$-family members themselves. Furthermore, even the descents and inversions due to the inhabitants of the last component among themselves remain fixed, since only the relative sizes matter. In addition the sum of the contributions to the weights from the relative placements of the members of the $V_{r}$-family within the first $c-1$ components remain the same, regardless the occupants of the last component. (Only relative size matters.) The only things that do change are the inversions caused by the interaction between the $V_{r}$-members that occupy the last component and the rest of their family. If the set of occupants of the last component is (let $\alpha_{c}=L$ ) $\left(x_{1}, \ldots, x_{L}\right) \quad\left(x_{1}<x_{2}<\cdots<x_{L}\right)$, then the number of inversions contributed by these interactions is $\left(d-\bar{x}_{L}\right)+\left(d-1-\bar{x}_{L-1}\right)+\cdots\left(d-(L-1)-\bar{x}_{1}\right)$, where $\bar{x}_{i}$ are the reduced labels in the $V_{r}$-family: $\bar{x}_{i}:=x_{i}-r d$. What we get is a typical partition with $\leq L$ parts and largest part $\leq d-L$, whose generating function (see [An, Chapter 3]) is $\left(1-q^{d}\right) \cdots\left(1-q^{\bar{d}-L+1}\right) /(1-q) \cdots\left(1-q^{L}\right)$, and therefore vanishes when $q=\omega$. Since all the unhappy seatings can be partitioned into these sets, they all die. 
Note. The same argument exactly goes over to prove Theorem 4 of the present paper. We partition each color into the appropriate number of families and "bachelors", and in order to kill the unhappy seatings, we choose the lowest color that has an unhappy family, and within this color, the lowest unhappy family.

TABLES OF $A_{n_{1}, n_{2}, n_{3}}\left(s ; q_{1}, q_{2}, q_{3}\right)$ FOR $6 \geq n_{1} \geq n_{2} \geq n_{3} \geq 0$

A table of the polynomials $A_{n_{1}, n_{2}, n_{3}}\left(s ; q_{1}, q_{2}, q_{3}\right)$ such that $n_{2}=0,1$ and $n_{3}=0,1$ and $n_{1} \leq 6$ has already been published by Désarménien [De]. Write $A_{m, n}^{d}$ for the notatons adopted in [De] and keep $A_{n_{1}, n_{2}, n_{3}}$ for ours. We have the correspondence $A_{n_{1}, n_{2}, n_{3}}=A_{n_{2}+n_{3}, n_{1}}^{d}$, whenever $0 \leq n_{2}, n_{3} \leq 1$. We then just list $A_{2,2,0}, A_{3,2,0}, A_{2,2,1}, A_{4,2,0}, A_{3,3,0}, A_{3,2,1}, A_{2,2,2}$ that have not appeared before. Only the last one actually involves the four variables $s, q_{1}, q_{2}, q_{3}$.

For example, the $(2,2,0)$-table is to be read: $A_{2,2,2}\left(s ; q_{1}, q_{2}, q_{3}\right)=s+$ $s^{2}\left(3+3 q_{1}+3 q_{2}+q_{1} q_{2}\right)+s^{3}\left(1+3 q_{1}+3 q_{2}+4 q_{1} q_{2}\right)+s^{4}$.

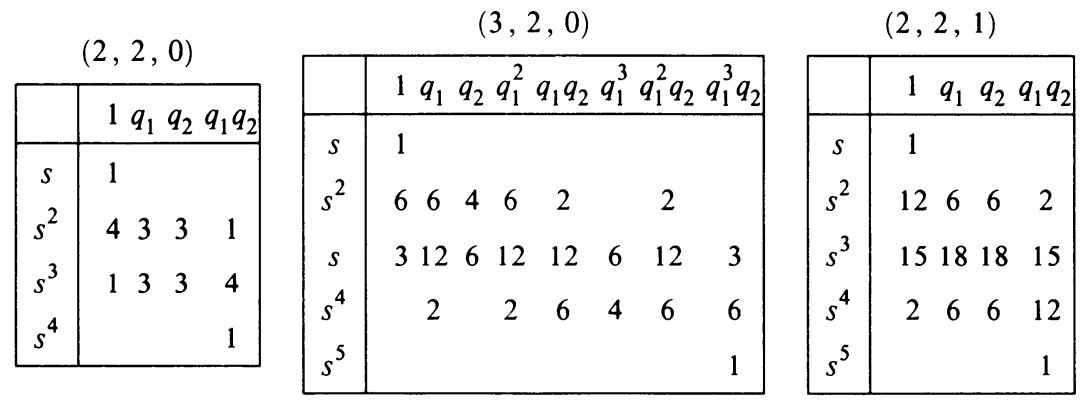

$(4,2,0)$

\begin{tabular}{|l|cccccccccccccc|}
\hline & \multicolumn{1}{|c|}{$q_{1}$} & $q_{2}$ & $q_{1}^{2}$ & $q_{1} q_{2}$ & $q_{1}^{3}$ & $q_{1}^{2} q_{2}$ & $q_{1}^{4}$ & $q_{1}^{3} q_{2}$ & $q_{1}^{5}$ & $q_{1}^{4} q_{2}$ & $q_{1}^{6}$ & $q_{1}^{5} q_{2}$ & $q_{1}^{6} q_{2}$ \\
\hline$s$ & 1 & & & & & & & & & & & & & \\
$s^{2}$ & 8 & 9 & 5 & 12 & 3 & 9 & 4 & 3 & 3 & & 1 & & & \\
$s^{3}$ & 6 & 27 & 10 & 42 & 24 & 45 & 35 & 33 & 33 & 18 & 20 & & 9 & \\
$s^{4}$ & & 9 & 20 & 18 & 33 & 33 & 35 & 45 & 24 & 42 & 10 & 27 & 6 \\
$s^{5}$ & & & 1 & & 3 & 3 & 4 & 9 & 3 & 12 & 5 & 9 & 8 \\
$s^{6}$ & & & & & & & & & & & & & 1 \\
\hline
\end{tabular}


$(3,3,0)$

\begin{tabular}{|c|c|c|c|c|c|c|c|c|c|c|c|c|}
\hline & $1 q_{1} q_{2} q_{1}^{2}$ & $q_{1} q_{2}$ & $q_{2}^{2} q_{1}^{3}$ & $q_{1}^{2} q_{2}$ & $q_{1} q_{2}^{2}$ & $q_{2}^{3}$ & $q_{1}^{3} q_{2}$ & $q_{1}^{2} q_{2}^{2}$ & $q_{1} q_{2}^{3}$ & $q_{1}^{3} q_{2}^{2}$ & $q_{1}^{2} q_{2}^{3}$ & $q_{1}^{3} q_{2}^{3}$ \\
\hline$s$ & 1 & & & & & & & & & & & \\
\hline$s^{2}$ & $\begin{array}{llll}9 & 8 & 8 & 8\end{array}$ & 4 & 8 & 4 & 4 & & & 4 & & & & \\
\hline$s^{3}$ & 9242424 & 36 & 2410 & 36 & 36 & 10 & 8 & 36 & 8 & 8 & 8 & 1 \\
\hline$s^{4}$ & 1888 & 36 & $\begin{array}{ll}8 & 10\end{array}$ & 36 & 36 & 10 & 24 & 36 & 24 & 24 & 24 & 9 \\
\hline$s^{5}$ & & 4 & & 4 & 4 & & 8 & 4 & 8 & 8 & 8 & 9 \\
\hline$s^{6}$ & & & & & & & & & & & & 1 \\
\hline
\end{tabular}

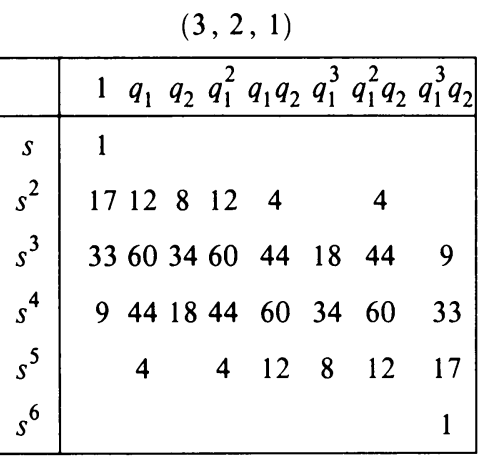

$$
(2,2,2)
$$

\begin{tabular}{|c|cccccccc|}
\hline & \multicolumn{1}{|c|}{$q_{1}$} & $q_{2}$ & $q_{3}$ & $q_{1} q_{2}$ & $q_{1} q_{3}$ & $q_{2} q_{3}$ & $q_{1} q_{2} q_{3}$ \\
\hline$s$ & 1 & & & & & & & \\
$s^{2}$ & 20 & 9 & 9 & 9 & 4 & 3 & 3 & 1 \\
$s^{3}$ & 48 & 45 & 45 & 45 & 33 & 33 & 33 & 20 \\
$s^{4}$ & 20 & 33 & 33 & 33 & 45 & 45 & 45 & 48 \\
$s^{5}$ & 1 & 3 & 3 & 3 & 9 & 9 & 9 & 20 \\
$s^{6}$ & & & & & & & & 1 \\
\hline
\end{tabular}

\section{REFERENCES}

[An] George E. Andrews, The theory of partitions, Encyclopedia of Math and its Appl., vol. 2, Addison-Wesley, Reading, Mass., 1976.

[BjWa] Anders Björner and Michelle L. Wachs, Permutation statistics and linear extensions of posets, 1988.

[De] Jacques Désarménien, Fonctions symétriques associées à des suites classiques de nombres, Ann. Sci. Ećole Norm. Sup. 16 (1983), 271-304.

[DeFo1] Jacques Désarménien and Dominique Foata, Fonctions symétriques et séries hypergéométriques basiques multivarées, Bull. Soc. France 113 (1985), 3-22.

[DeFo2] _ _ Statistiques d'ordre sur les permutations colorées, Discrete Math. 87 (1991), 133-148.

[DeFo3] _ _ The signed Eulerian numbers (submitted).

[Fo] Dominique Foata, On the Netto inversion number of a sequence, Proc. Amer. Math. Soc. 19 (1968), 236-240.

[Fo-Sch] Dominique Foata and Marcel-Paul Schützenberger, Major index and inversion of permutations, Math. Nachr. 83 (1978), 143-159.

[Ga] George Gasper, Summation, transformation, and expansion formulas for bibasic series, Trans. Amer. Math. Soc. 312 (1989), 257-277.

[GaRa1] George Gasper and Mizan Rahman, An indefinite bibasic summation formula and some quadratic, cubic, and quartic summation and transformation formulas, Canad. J. Math. 42 (1990), 1-27.

[GaRa2] __ Basic hypergeometric series, Cambridge Univ. Press (to appear).

[Ge1] Ira Gessel, Generating functions and enumeration of sequences, Ph. D. thesis, Dept. Math., M.I.T., Cambridge, Mass., 1977, pp. 111.

[Ge2] A q-analogue of the exponential formula, Discrete Math. 40 (1982), 69-80.

[Kn] Donald E. Knuth, The art of computer programming, vol, 3, Sorting and Searching, Addison-Wesley, Reading, Mass., 1973. 
[Lod] Jean-Louis Loday, Opérations sur l'homologie cyclique des algèbres commutatives, Invent. Math. 96 (1989), 205-230.

[Lot] M. Lothaire, Combinatorics on words, Encyclopedia of Math. and its Appl., vol. 17, Addison-Wesley, Reading, Mass., 1983.

[Mac] I. G. MacDonald, Symmetric functions and Hall polynomials, Clarendon Press, Oxford, 1979.

[Re] J. B. Remmel, Permutation statistics and $(k, l)$-hook Schur functions, Discrete Math. 67 (1987), 271-298.

[Ri] John Riordan, An introduction to combinatorial analysis, Wiley, New York, 1958.

[St] Richard P. Stanley, Binomial posets, Möbius inversion, and permutation enumeration, J. Combin. Theory Ser. A 20 (1976), 336-356.

[Wa] Michelle J. Wachs, An involution for signed Eulerian numbers (submitted).

[Ze] Doron Zeilberger, A lattice walk approach to the counting of multiset permutations, J. Math. Anal. Appl. 74 (1980), 192-199.

Department of Mathematics, Drexel University, Philadelphia, Pennsylvania 19104

Current address, D. Foata: Département de Mathématique, Université Louis-Pasteur, 7, Rue René-Descartes, F-67084 Strasbourg, France

Current address, D. Zeilberger: Department of Mathematics, Temple University, Philadelphia, Pennsylvania 19122 